Bulletin of the Section of Logic

Volume 50/2 (2021), pp. 151-176

https://doi.org/10.18778/0138-0680.2021.05

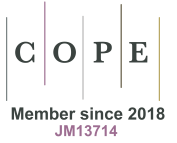

Daniela Glavaničová

Matteo Pascucci

\title{
THE GOOD, THE BAD, AND THE RIGHT: FORMAL REDUCTIONS AMONG DEONTIC CONCEPTS $^{1}$
}

\begin{abstract}
The present article provides a taxonomic analysis of bimodal logics of normative ideality and normative awfulness, two notions whose meaning is here explained in terms of the moral values pursued by a given community. Furthermore, the article addresses the traditional problem of a reduction among deontic concepts: we explore the possibility of defining other relevant normative notions, such as obligation, explicit permission and Hohfeldian relations, in terms of ideality and awfulness. Some proposals in this respect, which have been formulated in the literature over the years, are here improved and discussed with reference to the various logics that we will introduce.
\end{abstract}

Keywords: Awfulness, explicit permission, Hohfeldian relations, ideality, moral values, obligation.

2020 Mathematical Subject Classification: 03B45, 03B60, 03B80.

${ }^{1}$ Daniela Glavaničová was supported by the Slovak Research and Development Agency under the contract no. APVV-17-0057, VEGA 1/0197/20, and VEGA 2/0117/19. Matteo Pascucci was supported by the Štefan Schwarz Fund for the project "A fine-grained analysis of Hohfeldian concepts" and by the VEGA grant no. 2/0117/19.

Presented by: Tomasz Jarmużek, Fengkui Ju, Piotr Kulicki, Beishui Liao

Received: February 11, 2020

Published online: April 1, 2021

(C) Copyright by Author(s), Łódź 2021

(C) Copyright for this edition by Uniwersytet Łódzki, Łódź 2021 


\section{Moral values and moral principles: a philosophical preamble}

There are many sorts of concepts that play an important role in normative reasoning, some of which have been extensively analysed also within formal logic: obligation, prohibition, and permission; right, duty, privilege, power, and immunity; responsibility, liability, blame and praise; good, bad, supererogatory, ideal, awful, and so forth (see [8] for a survey). In the present paper, we will assume that the range of application of these notions is not absolute, but relative to the moral values and normative systems of a given community; however, this is not a necessary stance. ${ }^{2}$ To give an example, blame depends on actions of normative parties (individuals or groups), as well as on prospective responsibilities they had at the time of acting (or not acting, for that matter); see [3]. Our work focuses on the notions of normative ideality and awfulness, which are related to what is taken to be "the (highest) good" and "the (lowest) bad", respectively. Arguably, these two notions ultimately depend on the values of a given community: an industrialized country will likely have a different ordering of moral values than a small isolated tribe, and, consequently, they will have a different understanding of the notions of ideality and awfulness. Therefore, here normative ideality is equated with "what is ideal according to the moral values supported by a given community" and normative awfulness is equated with "what is awful according to the moral values supported by a given community".

Of course, a lot has been said about moral values, and they have been understood in different ways. To begin with, it is difficult to distinguish moral values from values of other kind. As Quine puts it in [15], p. 473:

There are easy extremes: the value that one places on his neighbor's welfare is moral, and the value of peanut brittle is not. The value of decency in speech and dress is moral or ethical in the etymological sense, resting as it does on social custom;

\footnotetext{
${ }^{2}$ We would also like to note that our assumption leaves it open how large overlaps there are between the moral values of different communities and to which extent these overlaps are relevant when one defines ideality and awfulness. For instance, if one generates the sets of propositions $P_{1}, P_{2}, P_{3}, \ldots$ that hold if the norms of communities $c_{1}, c_{2}, c_{3}, \ldots$ are respectively met, one can say that the propositions that are in the set $P=P_{1} \cap P_{2} \cap P_{3} \cap \ldots$ (that is, the propositions that are in the "common base") are ideal absolutely. A similar idea can be employed for the notion of awfulness.
} 
and similarly for observance of the Jewish dietary laws. On the other hand the eschewing of unrefrigerated oysters in the summer, though it is likewise a renunciation of immediate fleshly pleasure, is a case rather of prudence than morality.

Now, the question is: to which extent can moral values and principles be the objects of a formal analysis? More often than not, moral values are understood as notions of fairness, justice, trust, respect, responsibility, privacy, sharing, loyalty, and so forth (see, for instance [16], [17], and [19]). These, if taken straightforwardly, are neither truth-apt, nor can be consistent or inconsistent, nor have consequences. However, it is also very common to equate moral values with the corresponding moral principles or norms (for instance, trying to "teach" moral values to a robot may mean providing it with instructions encoding certain moral principles). We will follow the former common practice and occasionally speak about moral values understood as the corresponding principles.

Moral principles, by contrast, can be regarded as propositions. When moral values are equated with these, they become truth-apt, can have consequences and be either consistent or inconsistent (for instance, the moral value of fairness can be transformed into the moral principle "according to our moral values, everyone is treated fairly"). Naturally, this is just one option how to respond to a variant of Jørgensen dilemma; see [12]. Perceived in this manner, the value of sharing information can go against the value of privacy (see [19]), and the value of loyalty to an authority (like a family member) can very well go against the values of justice and fairness.

Another preliminary remark on the philosophical ground that will be used to support our work is needed: the notion of ideality employed in the present paper is taken to an extreme, since it describes (portions of) perfect situations (all moral values of a given community are realised, or more colloquially, all good things are done). This approach follows certain semantic intuitions at the basis of the traditional interpretation of deontic logic, as discussed in [11]. Similarly, the notion of awfulness employed here describes (portions of) the worst situations possible (no moral values of a given community are realised, or more colloquially, no good things are done). To better illustrate this point, it is useful to offer a comparison with how the notions of ideality and awfulness are used in everyday reasoning: we ordinarily regard a scenario as awful as soon as one terrible thing blatantly against the accepted moral values occurs (e.g., a situation where, 
within a group of accused people, one innocent person is convicted, while all the others are judged in an appropriate way). Similarly, it is common to say that a situation where some relevant set of great things happens is ideal (for instance, when there is perfect justice with respect to important issues), even though other, less relevant, bad things happen (people are occasionally dishonest in minor issues).

In the present article we will provide a taxonomy of bimodal logics to represent the notions of normative ideality and normative awfulness; furthermore, we will assess ideas to formally define other relevant normative notions in terms of these two. In particular, we will examine the case of obligation, of explicit permission, and of some Hohfeldian concepts, improving and extending some proposals available in the literature.

\section{Related approaches}

A natural starting point of our work is a logical system called DL introduced by A. Jones and I. Pörn in [11] in order to represent deductive reasoning with normative ideality and normative sub-ideality. The aim of DL is to address some of the criticisms traditionally raised against what is known as the standard system of deontic logic, namely SDL. The latter is a variant of the alethic modal system $\mathbf{K D}$ based on a language with a primitive operator of obligation, $O{ }^{3}$ Jones and Pörn claim that the main problem of SDL is a semantic one: the meaning of the operator of obligation is explained in terms of a set of normatively ideal situations (or worlds), according to the following truth-conditions, where $\phi$ is a formula denoting an arbitrary proposition, $w$ an arbitrary situation and "iff" abbreviates "if and only if":

- $O \phi$ holds in $w$ iff $\phi$ holds in every situation $v$ that is normatively ideal with respect to $w$.

The argument for their criticism is very simple: what is obligatory cannot be equated with what holds in all normatively ideal situations, since tautologous propositions (e.g., that either it rains or it does not rain) clearly hold in all such situations, while being normally regarded as normatively neutral (that is, as neither obligatory, nor forbidden, nor permitted). In order for

\footnotetext{
${ }^{3}$ For the criteria followed in the present article for naming alethic modal systems, see, e.g., [4].
} 
a proposition to represent an obligation, there must be some normatively sub-ideal situation in which it fails to hold. ${ }^{4}$

Here we will adopt this view for the sake of exploring its formal consequences, while noting also that the question whether tautologous propositions are permitted heavily depends on how one reads the term "permitted". If the permission of $\phi$ is merely the lack of an obligation of $\neg \phi$ (i.e., what is sometimes referred to as an implicit permission), then it is plausible to say that tautologies are permitted. Natural languages, however, suggest stronger readings of "permitted", such as explicit permission or free choice permission. Explicit permission corresponds to a permission explicitly given; free choice permission, in turn, corresponds to a permission that allows one to infer " $\phi$ is permitted" and " $\psi$ is permitted" from " $\phi \vee \psi$ is permitted"; see [6] on the main notions of permission we use in natural languages.

Even if one does not engage with truth conditions for $O \phi$ that involve a complete description of normatively ideal situations, what is ideal with respect to the moral values of a certain community need not correspond to what is obligatory with respect to these values. Nevertheless, the former has a certain impact on the latter. For instance, if a community aims at an equal distribution of the goods available, this has consequences on what an individual is expected to do; however, no single individual is expected to produce on her own a situation in which all goods are equally distributed: therefore, the latter situation is normatively ideal according to the moral values of the community, while not constituting directly an obligation for anybody. One is rather obliged to perform specific actions (or bring about states-of-affairs) that contribute to its realization.

Looking for a formal distinction between what is normatively ideal and what is obligatory, Jones and Pörn propose to adopt a language where, in the place of the operator $O$, there are two primitive modal operators that we will here represent as $\square$ and $\boldsymbol{\square}$. They suggest the following reading:

- $\square \phi$ holds in $w$ iff $\phi$ holds in every situation $v$ that is normatively ideal with respect to $w$;

- $\phi$ holds in $w$ iff $\phi$ holds in every situation $v$ that is normatively sub-ideal with respect to $w$.

\footnotetext{
${ }^{4}$ This problem is already pointed out in [1] by A. R. Anderson, who suggests a restriction of deontic discourse to contingent propositions.
} 
Therefore, $\square$ has in DL the same interpretation that $O$ has in SDL; however, it is said to be an operator for normative ideality, rather than for obligation. Furthermore, as the truth-conditions indicate, $\mathbf{\square}$ is said to be an operator for normative sub-ideality. Subsequently, they define an operator of obligation in the following manner (we will use the label Ob1 for this definition) $:^{5}$

\section{Ob1 $O \phi={ }_{\text {def }} \square \phi \wedge \neg \mathbf{\square} \phi$.}

The meaning of this definition is that $\phi$ is obligatory in a situation $w$ iff $\phi$ holds in all normatively ideal situations (with respect to $w$ ) and fails to hold in some normatively sub-ideal situations (with respect to $w$ ).

Jones and Pörn do not engage with the task of axiomatizing their logic, which is rather specified in terms of a list of valid formulas in a given class of relational models. They just observe that DL is at least as powerful as a bimodal copy of SDL. A complete axiomatization is indicated in [5]: one needs to extend a suitable basis for bimodal SDL with the axiom-schema $(\square \phi \wedge \square) \rightarrow \phi$, which captures the idea that the current situation is either ideal or sub-ideal.

The idea of defining $O$ in terms of $\square$ and $\boldsymbol{\square}$ as in Ob1 looks like a simple and elegant solution; however, it encounters some obstacles when one attempts to deal with deontic paradoxes, as well as to represent contraryto-duty reasoning (see the discussion in [14] and [13]). The move from a formal analysis of ideality and sub-ideality to a formal analysis of ideality and awfulness is suggested by an alternative definition of $O$ in terms of the same bimodal language that can be found in [5]:

Ob2 $O \phi={ }_{\text {def }} \square \phi \wedge \boldsymbol{\square} \neg \phi$.

On the one hand, $\mathrm{Ob} 2$ is able to overcome some of the problems of $\mathrm{Ob} 1$, (for instance, by solving some traditional deontic paradoxes). On the other hand, it cannot be easily reconciled with the reading of $\mathbf{\square}$ as "in all normatively sub-ideal situations": even if $\phi$ is obligatory, it can very well hold in a sub-ideal situation (and thus there can be a sub-ideal situation where $\neg \phi$ does not hold). This is possible because a situation can be sub-ideal due to a violation of some other obligation than $\phi$. For example, if everyone in a village pays their debts $(\phi)$, the situation will still be sub-ideal

\footnotetext{
${ }^{5}$ We point out that there are some relevant notational differences between this article and [11]: for instance, there $O$ stands for normative ideality, $O^{\prime}$ for normative subideality, and Ought for obligation.
} 
if there is a serial killer murdering people in the neighbourhood. Thus, in [13] it is suggested to adopt $\mathrm{Ob} 2$ and, at the same time, change the reading of to "in all normatively awful situations". Such a move allows one to get rid of the axiom $(\square \phi \wedge \boldsymbol{\square}) \rightarrow \phi$, since the current situation (i.e., the situation in which things are evaluated) might be neither ideal nor awful. Furthermore, explicit permission is defined in [13] as follows, adhering to the original proposal in [11] (though, under a different reading of $\downarrow$, which shortens $\neg \square$ ):

\section{Pm1 $P \phi=_{\text {def }} \diamond \phi \wedge \neg \neg \phi$.}

Contrary to SDL, the operator of explicit permission in this case is not the dual of the operator of obligation.

\section{A general representation of conditional norms}

A comprehensive appraisal of the results obtained so far within attempts to reduce deontic concepts to normative ideality and related notions shows several limitations. For instance, it seems that approaches of this kind need additional devices (e.g., reference to levels of ideality) for a proper treatment of contrary-to-duty reasoning. Nevertheless, apart from these limitations, several aspects of the reductionist project have just not been addressed yet. For instance, consider the problem of defining simple conditional obligations, like " $\phi$ is obligatory under condition $\psi$ ", in terms of ideality and awfulness. According to $\mathrm{Ob} 2$, one could say that each of these obligations corresponds to a formula of the kind $\square(\psi \rightarrow \phi) \wedge \boldsymbol{\square} \neg(\psi \rightarrow \phi)$, which constitutes the definiens of $O(\psi \rightarrow \phi)$. Yet, this approach is not satisfactory, since in many logical systems it commits one to the claim that the antecedent $\psi$ holds in every normatively awful situation and the consequent $\phi$ in none: in fact, as soon as it is possible to replace formulas that are provably equivalent in the Propositional Calculus within the scope of $\boldsymbol{\square}$, one is entitled to infer $\mathbf{\square}(\psi \wedge \neg \phi)$ from $\boldsymbol{\square} \neg(\psi \rightarrow \phi)$.

Here we propose to improve the solutions available by adopting a general definition that applies both to conditional and unconditional obligations. First, we generalize the meaning of $\square$ and $\boldsymbol{\square}$, in order to avoid making reference to a complete description of the set of normatively ideal situations and the set of normatively awful situations, differently from [11]. This is due to the fact that reference to complete descriptions of ideal/awful situations 
commits one to certain forms of logical inference that are not available in the weakest systems that we are going to introduce. For instance, if no other restriction is specified, when one says that in all normative ideal situations $\phi$ is the case, then one is very likely committed to say that in all such situations $\phi \vee \psi$ is the case too. Thus, in order to avoid similar inferences, a different and more general reading of $\square$ and $\boldsymbol{\square}$ is needed. ${ }^{6}$ We will say that $\square \phi$ means that $\phi$ is a consequence of the fact that all the moral values of a given community are pursued, and that $\phi$ means that $\phi$ is a consequence of the fact that no moral values of a given community are pursued. The notion of consequence here involved is intentionally left unspecified; indeed, as we will see, the plausibility of a more precise characterization will depend on the formal system analysed. Accordingly, the intended interpretation of $\diamond \phi$ (which shortens $\neg \square \neg \phi$ ), is that $\phi$ is compat$i b l e$ with the fact that all moral values of a given community are pursued; the intended interpretation of $\phi$ is that $\phi$ is compatible with the fact that no moral value of a given community is pursued.

Conditional obligations will be taken to represent the most general case, and unconditional obligations will be defined in terms of them. More precisely, an unconditional obligation $O \phi$ will be treated as a shorthand for an obligation trivially depending on a tautologous condition, as it is often done in the literature on dyadic deontic logic (see, e.g., [2]), and will be represented as $O(\phi / \top)$. The general definitional schema that we will adopt is the following:

$$
\mathrm{Ob} * O(\phi / \psi)=_{\text {def }} \diamond \psi \wedge \square(\psi \rightarrow \phi) \wedge(\diamond \psi \rightarrow \neg \diamond(\psi \wedge \phi)) .
$$

The meaning of $\mathrm{Ob} *$ is that $\phi$ is obligatory under condition $\psi$ if and only if (i) $\psi$ is compatible with normative ideality, (ii) it is normatively ideal that $\psi$ entails $\phi$, and (iii) if $\psi$ is compatible with normative awfulness, then the conjunction of $\psi$ and $\phi$ is incompatible with normative awfulness. Thus, in the case of unconditional obligations we will get the definiens: $\diamond \top \wedge \square(T \rightarrow$ $\phi) \wedge(\checkmark \top \rightarrow \neg(\top \wedge \phi))$. We will see that in many classes of systems such a definiens can be simplified. Looking at the range of application of $\mathrm{Ob} *$, we

\footnotetext{
${ }^{6}$ For a parallel, consider the inadequacy (in general) of reading the modal operator of alethic necessity as "it is the case in all possible worlds that" when one wants do deal with classes of systems weaker than $\mathbf{K}$, especially systems not closed under the replacement of provable equivalents. Also in this case, a broader reading of the operator at issue is needed.
} 
need to clarify that this definition works for conditional obligations that do not instantiate a form of contrary-to-duty reasoning. ${ }^{7}$

Moreover, we can define implicit permission in terms of $\mathrm{Ob} *$ and negation:

$\operatorname{IPm} * \neg O(\neg \phi / \psi)=_{\text {def }} \neg((\diamond \psi \wedge \square(\psi \rightarrow \neg \phi)) \wedge(\diamond \psi \rightarrow \neg \vee(\psi \wedge \neg \phi)))$.

In systems where replacement of formulas that are provably equivalent in the Propositional Calculus is available (we will later call this $\operatorname{RRPE}_{P C}$ ), the above definiens can be further transformed, so as to get the schema $(\diamond \psi \rightarrow \diamond(\psi \wedge \phi)) \vee(\diamond \psi \wedge \diamond(\psi \wedge \neg \phi))$. The latter reads as follows: if the antecedent is compatible with normative ideality, then the antecedent and the consequent are jointly compatible with normative ideality; otherwise the antecedent is compatible with normative awfulness and so are, jointly, the antecedent and the negation of the consequent. The first disjunct of this simplified definiens appears plausible with respect to the intended interpretation: if our moral values allow for the condition of a permission to hold, so they allow for this condition along with the permitted formula. The second disjunct can be justified with respect to the intended interpretation too: the condition is compatible with awfulness and so is the condition along with the negation of the permitted formula. A further look at the role played by $\checkmark(\psi \wedge \neg \phi)$ : for example, one does not get tested for coronavirus even though satisfying all conditions for getting tested, then getting tested for coronavirus (when satisfying all conditions) cannot be prohibited. This issue connects to the intuition that what is not prohibited is implicitly permitted.

By contrast, explicit permission can be defined as follows:

$\operatorname{EPm} * P(\phi / \psi)=_{\text {def }}(\diamond \psi \rightarrow \diamond(\psi \wedge \phi)) \wedge(\diamond \neg \psi \vee \diamond(\psi \wedge \neg \phi))$

${ }^{7}$ As we mentioned in section 2 , the representation of contrary-to-duty norms is a very challenging issue in a framework for normative ideality and normative awfulness, and it very likely requires the addition of levels of ideality. This problem, pointed out also by a reviewer, is left open for future research. The reason why $\mathrm{Ob} *$ is not adequate in this regard is that its "ideality component", that is, the conjunction $\diamond \psi \wedge \square(\psi \rightarrow \phi)$, commits one to the claim that $\psi$ is compatible with normative ideality, whereas this is not the case in contrary-to-duty reasoning. However, even weakening this component to $\diamond \psi \rightarrow \square(\psi \rightarrow \phi)$ seems to be problematic, because one loses the dependence of $\phi$ from $\psi$ in ideal situations (namely, the truth of $\square(\psi \rightarrow \phi)$ ) in cases in which $\nabla \psi$ does not hold. 
The reading of $\mathrm{EPm} *$ is: " $\phi$ is permitted under condition $\psi$ iff (i) if $\psi$ is compatible with normative ideality, then so is $\psi \wedge \phi$, and (ii) $\neg \psi$ is compatible with normative awfulness, or $\psi \wedge \neg \phi$ is compatible with normative awfulness." Unconditional explicit permissions are then defined as follows, exploiting the usual strategy of equating $P \phi$ with the conditional permission $P(\phi / T):(\diamond \top \rightarrow \diamond(T \wedge \phi)) \wedge(\neg \neg \vee \wedge(\top \wedge \neg \phi))$. Notice that, in general, explicit permission entails implicit permission (due to laws of the Propositional Calculus) but not vice versa. In this article we will mainly focus on obligations. Furthermore, we will explore a variation of the bimodal language with indexed operators in which it is possible to express basic Hohfeldian concepts, such as duties and rights, involving two normative parties $[9,10]$.

\section{Formal language}

In the present exposition we will extend the bimodal language used in [11] with a propositional constant $\mathfrak{c}$, called ideality witness and meaning "all moral values of the community are pursued". This constant will allow us to ensure that the description of what is normatively ideal and the description of what is normatively awful according to a system are always distinct, unless the set of moral values gives rise to inconsistencies on its own in a given situation. Our language will be simply called $\mathcal{L}$.

Definition 4.1 (Vocabulary). The language $\mathcal{L}$ includes the following primitive symbols:

- a countable set of propositional variables Var, denoted by $p, q, r$, etc.;

- the propositional constant $\mathfrak{c}$ (ideality witness);

- the modal operators $\square$ (normative ideality) and $\boldsymbol{\square}$ (normative awfulness);

- the Boolean connectives $\neg$ (negation) and $\rightarrow$ (material implication);

- round brackets. 
Definition 4.2 (Well-formed formulas). The set WFF of well-formed formulas over $\mathcal{L}$ is defined by the grammar below, where $p \in \operatorname{Var}$ :

$$
\phi::=p|\mathfrak{c}| \neg \phi|\phi \rightarrow \phi| \square \phi \mid \mathbf{\square} \phi
$$

Additional Boolean operators $(\wedge, \vee$ and $\equiv)$ and modal operators $(\diamond$ and $\checkmark)$ can be defined in terms of the primitive ones according to the usual conventions. For instance, $\diamond \phi={ }_{\text {def }} \neg \square \neg \phi$. We adopt standard conventions also for the definition of the logical constants $\top$ (verum) and $\perp$ (falsum). Furthermore, we take the dyadic operators $O$ (obligation) and $P$ (explicit permission) to be defined in accordance with Ob* and EPm*. We use $\operatorname{Var}(\phi)$ to denote the set of propositional variables having some occurrences in $\phi$. A formula $\phi$ is a substitution instance of a formula $\psi$ iff $\phi$ is obtained from $\psi$ by uniformly substituting the occurrences of some elements of $\operatorname{Var}(\psi)$ with a possibly different formula. For instance, $(\square r \rightarrow q) \rightarrow(\square r \rightarrow q)$ is a substitution instance of $p \rightarrow p$. Furthermore, trivially, according to this definition, every formula is a substitution instance of itself. We will denote by $\mathrm{WFF}^{b}$ the subset of WFF including all formulas with no occurrence of a modal operator (i.e., the set of purely Boolean formulas).

\section{Deductive systems}

In this section we will describe a series of nine modal systems that can be used for formal reasoning on the notions of normative ideality and normative awfulness. Our aim is giving some examples of a wide range of logical possibilities that can be exploited for various applications. Furthermore, we will see how the definitions of obligation and explicit permission mentioned in the previous part of the article, namely $\mathrm{Ob} *$ and EPm*, behave within these systems. First, we introduce some preliminary notions that will be used in the axiomatic bases of these systems. We will denote the classical Propositional Calculus as $P C$. The symbol $\vdash$ will indicate derivability in a system (that can be either specified by the context, or arbitrary); the symbol $\vdash_{P C}$ derivability in $P C$. We start with the notion of a transformation group, which will be used to define a form of restricted replacement for provable equivalents.

Definition 5.1 (Transformation group). A transformation group is a set of formulas $g=\left\{\phi_{1}, \phi_{2}, \phi_{3}, \ldots\right\}$ where, for $1 \leq i, j$, we have that $\phi_{i}, \phi_{j} \in$ WFF $^{b}$ and $\vdash_{P C} \phi_{i} \equiv \phi_{j}$. 
Transformation groups thus concern only purely Boolean formulas. We will use $\mathcal{C}$ to denote a set of transformation groups. Examples of transformation groups are $\{p, \neg \neg p\},\{p \wedge q, q \wedge p,(p \wedge q) \wedge(q \wedge p)\},\{\neg(\neg p \wedge \neg q), p \vee q, \neg p \rightarrow q\}$, $\{q \rightarrow q, r \rightarrow r\}$, etc. We will restrict our attention to transformation groups in which all formulas are distinct, and to sets of transformation groups where every formula occurs at most in one group.

Furthermore, we need to introduce a notion of analogous substitution on which we will rely, in combination with transformation groups, when defining an axiom of Modal Dependence.

Definition 5.2 (Analogous substitution instances). Formulas $\phi_{1}, \ldots, \phi_{n}$ are said to be analogous substitution instances of formulas $\psi_{1}, \ldots, \psi_{n}$ iff (i) $\phi_{i}$, for $1 \leq i \leq n$, is a substitution instance of $\psi_{i}$, and (ii) any propositional variable occurring in $\psi_{1}, \ldots, \psi_{n}$ is substituted by the same formula in $\phi_{1}, \ldots, \phi_{n}$.

Finally, we provide a standard notion of mirror-relation between two formulas of a bimodal language.

Definition 5.3 (Mirror image). The mirror image of a formula $\phi$, denoted by $\operatorname{mi}(\phi)$, is the result of replacing in $\phi$ each occurrence of an "ideality" modal operator with an occurrence of the corresponding "awfulness" modal operator, and vice versa.

For instance, $\operatorname{mi}(\diamond p \rightarrow \square q)=\nabla_{p} \rightarrow \square q$. An immediate consequence of Definition 5.3 is that, for every $\phi \in \mathrm{WFF}, \operatorname{mi}(\operatorname{mi}(\phi))=\phi$.

Next, we provide a list of deductive principles that will be taken into account in the axiomatic bases of the logical systems discussed in the present article. We will call such a list $\Theta$. All principles in $\Theta$ are either axioms or rules. $^{8}$ The first principle in $\Theta$ varies with one's choice of a set of transformation groups $\mathcal{C}$.

\footnotetext{
${ }^{8}$ We will speak of "axioms" with reference to principles that ultimately represent "axiom-schemata", as far as no ambiguity arises.
} 
$\mathrm{MD}_{\mathcal{C}} \quad \square \phi \rightarrow \square \phi^{\prime}$, provided that $\phi$ and $\phi^{\prime}$ are analogous substitution instances of two formulas $\psi$ and $\psi^{\prime}$, both occurring in one and the same transformation group $g \in \mathcal{C}$;

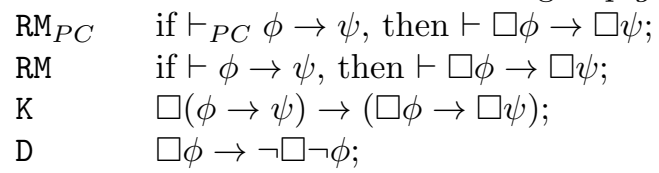

BR1 $\square \mathfrak{c} \wedge \boldsymbol{\square} \neg \mathfrak{c} ;$

BR2 $\square \mathbf{\square} \equiv \mathbf{\square} \phi$;

BR3 $\square \phi \equiv \square \phi$;

N $\quad \square T$;

$\mathrm{T}^{*} \quad \square(\square \phi \rightarrow \phi)$;

$4 \quad \square \phi \rightarrow \square \square \phi$.

A brief remark on some labels used: $\mathrm{MD}_{\mathcal{C}}$ denotes an axiom-schema for Modal Dependence modulo a set of transformation groups $\mathcal{C}$; $\mathrm{RM}_{P C}$ denotes monotony of the operator $\square$ with respect to provable implication in the Propositional Calculus. Rule RM denotes monotony of $\square$ without restrictions. $\mathrm{K}, \mathrm{D}, \mathrm{N}, \mathrm{T}^{*}$ and 4 are standard modal axioms. BR1, BR2 and BR3 denote various bridge-axioms connecting ideality and awfulness. Axiom BR1 says that the constant $\mathfrak{c}$ always distinguishes what is normatively ideal from what is normatively awful. Axiom BR2 says that "ideal awfulness" collapses to awfulness (this can be understood as a meta-level approval of what the community morally disapproves; if, according to our moral values, it is good that the slavery is wrong, then according to our values, slavery is wrong). Similarly, axiom BR3 says that "awful ideality" collapses to ideality (this axiom can be understood as a meta-level disapproval of what the community morally approves; for instance, a butcher from a future world can say that it is awful that a community morally approves a vegetarian diet only, and this would be naturally read as implying that the community does so).

If one allows the set of transformation groups $\mathcal{C}$ to be infinite, then the rule $\mathrm{RE}_{P C}$ described below (which indicates congruence of the operator $\square$ with respect to provable equivalence in $P C$ ) can be obtained from $\mathrm{MD}_{\mathcal{C}}$ - for instance, by trivially defining $\mathcal{C}$ as the partition of $\mathrm{WFF}^{b}$ under provable equivalence in $P C$. However, imposing the restriction that $\mathcal{C}$ is a finite set, and that each transformation group within it is finite as well, can be seen as 
a way of simulating actual reasoning procedures applied by an agent with bounded rationality. Furthermore, $\mathrm{RE}_{P C}$ is clearly derivable in a system where either $\mathrm{RM}_{P C}$ or $\mathrm{RM}$ is available.

$$
\mathrm{RE}_{P C} \quad \text { if } \vdash_{P C} \phi \equiv \psi \text {, then } \vdash \square \phi \equiv \square \psi .
$$

Note also that, conversely, $\mathrm{MD}_{\mathcal{C}}$, for any choice of $\mathcal{C}$, can be derived in any system closed under $\mathrm{RE}_{P C}$.

With a little abuse of terminology, we will also speak of the mirror image of an axiom $\mathrm{X}$ and of a rule RX. Our notation for these will be $\mathrm{mi}(\mathrm{X})$ and $\mathrm{mi}(\mathrm{RX})$. The meaning of the former expression is that we replace all ideality operators explicitly mentioned in the general formulation of axiom $\mathrm{X}$ with the corresponding awfulness operators, and vice versa. For instance, $\operatorname{mi}\left(\mathrm{T}^{*}\right)=\mathbf{\square}(\mathbf{\square} \rightarrow \phi)$. The meaning of the latter expression is that we replace all ideality operators explicitly mentioned in the general formulation of RX with the corresponding awfulness operators, and vice versa. For instance, mi(RM) $=$ "if $\vdash \phi \rightarrow \psi$, then $\vdash \mathbf{\square} \phi \rightarrow \mathbf{\square} \psi$ ".

All systems to be developed here are extensions of $P C$ and are closed under Modus Ponens (denoted by MP); we take the latter to be formulated in a way which allows one to reason under assumptions:

MP from the set of assumptions $\{\phi, \phi \rightarrow \psi\}$ infer $\psi$.

A few informal remarks on the derivations used in this article: a derivation $\mathrm{D}$ will be a finite sequence of lines labelled with natural numbers $1, \ldots, n$, each including exactly one formula that either (i) is a hypothesis (we regard claims on the set $\mathcal{C}$ as hypotheses too), or (ii) is an instance of an axiom, or (iii) is obtained from other formulas in the previous lines by applying one of the rules available. Axioms and rules used in derivations will depend on the systems under analysis, which will be clarified by the context. When a line $l$ of a derivation includes a formula $\phi$ that is obtained only via applications of axioms and rules whose mirror images are available in the system, we can add a further line $l+1$ with the formula $\operatorname{mi}(\phi)$ and use " $l \times$ Mirror Images" as a justification.

Axiomatic bases of systems are here ordered sequences of deductive principles. For each axiomatic basis presented, some initial sub-sequence of deductive principles is closed under mirror images. 
Definition 5.4 (Pre-axiomatic basis). A pre-axiomatic basis for a system $S$ over the language $\mathcal{L}$ is an ordered list $\sigma=\left\langle X_{1}, \ldots, X_{n}\right\rangle$, where $X_{i} \in \Theta$, for $1 \leq i \leq n$.

Definition 5.5 (Axiomatic basis). Given a pre-axiomatic basis $\sigma$ for a system $S$, the result of putting the symbol o, called a mirror image bookmark, over one of the items in $\sigma$ is an axiomatic basis for $S$.

A mirror image bookmark occurring on top of an item $X_{i}$ in a list $\sigma$ says that all principles $X_{j}$ in $\sigma$, s.t. $1 \leq j \leq i$, are closed under mirror images.

Now we have all ingredients needed to introduce axiomatic bases of systems. The first group of systems that we are going to analyse ( $\alpha$-systems) allow for a very restricted form of $\square$-congruence and $\mathbf{\square}$-congruence: it applies only to pairs of formulas that are in a relation of analogous substitution with some pair of formulas in a transformation group of the set $\mathcal{C}$. In all of these systems ideality and awfulness are at least characterized as contrary notions, due to the fundamental axiom BR1.

Definition 5.6 (System $S_{\alpha 1}$ ). The axiomatic basis of system $S_{\alpha 1}$ is specified by the following ordered list of deductive principles: $\left\langle\mathrm{MD}_{\mathcal{C}}, \mathrm{BR} 1\right\rangle$.

For any choice of a finite set $\mathcal{C}, S_{\alpha 1}$ can be regarded as the minimal $\mathcal{C}$-based non-congruential system. In such a system it is possible to derive a rule of restricted replacement of provable equivalents, namely the rule $\mathrm{RRPE}_{\mathcal{C}}$ described below.

$\operatorname{RRPE}_{\mathcal{C}}$ if $\phi$ and $\psi$ are analogous substitution instances of two formulas $\phi^{\prime}$ and $\psi^{\prime}$ both occurring in one and the same transformation group $g \in \mathcal{C}$, and $\chi_{2}$ is obtained from $\chi_{1}$ by replacing some occurrence of $\phi$ with $\psi$, then $\vdash \chi_{1}$ entails $\vdash \chi_{2}$.

When $\mathrm{RE}_{P C}$ and $\mathrm{mi}\left(\mathrm{RE}_{P C}\right)$ are derivable (due to the way in which $\mathcal{C}$ is defined) one gets replacement for all formulas that are provably equivalent in $P C$, which we can denote as $\operatorname{RRPE}_{P C}$.

An example of deductive argument that can be represented within system $S_{\alpha 1}$ is the following, provided that $\mathcal{C}$ includes a transformation group where both $\neg r \rightarrow \neg s$ and $r \vee \neg s$, for some $r, s \in$ Var, occur: 
Ideally, if citizens are not tested for coronavirus with a negative result $(\neg p)$, they do not go to work $(\neg q)$. Therefore, ideally, either citizens. are tested for coronavirus with a negative result, or they do not go to work.

$\begin{array}{lll}1 & (\neg r \rightarrow \neg s),(r \vee \neg s) \in g, \text { for some } g \in \mathcal{C} & \text { Hyp. } \\ 2 & \square(\neg p \rightarrow \neg q) & \text { Hyp. } \\ 3 & \square(\neg p \rightarrow \neg q) \rightarrow \square(p \vee \neg q) & 1 \times \text { MD }_{\mathcal{C}} \\ 4 & \square(p \vee \neg q) & 2,3 \times \text { MP }\end{array}$

System $S_{\alpha 1}$ can be interpreted, for instance, in the $\mathcal{L}$-models described below.

Definition 5.7. An $\mathcal{L}$-model is a tuple $\mathfrak{M}=\left\langle W, \mathbb{C}, f, h_{1}, h_{2}, V\right\rangle$, where:

- $W$ is a set of possible worlds, or situations, denoted by $w, v, u$, etc.;

- $\mathbb{C}$ is a set of semantic contents, denoted by $c, d, e$, etc. ${ }^{9}$

- $f$ is a function mapping WFF to $\mathbb{C}$ s.t. $f(\phi)$ is the semantic content of $\phi$;

- $h_{1}$ is a function mapping $W$ to $\wp(\mathbb{C})$ s.t. $h_{1}(w)$ is the set of ideal semantic contents at $w$;

- $h_{2}$ is a function mapping $W$ to $\wp(\mathbb{C})$ s.t. $h_{2}(w)$ is the set of awful semantic contents at $w$;

- $V$ is a function mapping $\operatorname{Var} \cup\{\mathfrak{c}\}$ to $\wp(W)$ s.t. $V(x)$ is the valuation of $x .^{10}$

Truth-conditions with reference to a situation $w$ in an $\mathcal{L}$-model $\mathfrak{M}$ are as usual, except for the following clauses:

- $\mathfrak{M}, w \vDash \mathfrak{c}$ iff $w \in V(\mathfrak{c})$;

- $\mathfrak{M}, w \vDash \square \phi$ iff $f(\phi) \in h_{1}(w)$;

- $\mathfrak{M}, w \vDash \mathbf{\square}_{\phi}$ iff $f(\phi) \in h_{2}(w)$.

${ }^{9} \mathrm{~A}$ semantic content can be informally interpreted as a fine-grained meaning expressed by a formula, namely something more informative than the set of possible worlds where the formula turns out to be true.

${ }^{10}$ We use $x$ to denote a member of the set $\operatorname{Var} \cup\{\mathfrak{c}\}$. 
An $\mathcal{L}$-model $\mathfrak{M}$ for $S_{\alpha 1}$ needs to satisfy the following properties, for a given choice of $\mathcal{C}$ and any situation $w \in W$ :

- $f(\mathfrak{c}) \in h_{1}(w)$ and $f(\neg \mathfrak{c}) \in h_{2}(w)$;

- if $\phi$ and $\phi^{\prime}$ are analogous substitution instances of two formulas $\psi$ and $\psi^{\prime}$, both occurring in one and the same transformation group $g \in \mathcal{C}$, then $f(\phi) \in h_{i}(w)$ only if $f\left(\phi^{\prime}\right) \in h_{i}(w)$, for $i \in\{1,2\}$.

The soundness of $S_{\alpha 1}$ with respect to this class of models can be easily checked by looking at the correspondence between the deductive principles in its axiomatic basis and the list of model properties. The same holds for the other classes of models that will be presented later and the associated formal systems. We leave open the problem of building a completeness proof in terms of the various classes of $\mathcal{L}$-models.

Definition 5.8 (System $S_{\alpha 2}$ ). The axiomatic basis of system $S_{\alpha 2}$ is specified by the following ordered list of deductive principles: $\left\langle\mathrm{MD}_{\mathcal{C}}, \stackrel{\circ}{\mathrm{K}}, \mathrm{BR} 1\right\rangle$.

$S_{\alpha 1}$ is too weak to capture many relevant deductive inferences. For instance, in $S_{\alpha 2}$, but not in $S_{\alpha 1}$, it is possible to represent arguments like the following, under the assumption that $\mathcal{C}$ includes a transformation group where both $r \rightarrow s$ and $\neg s \rightarrow \neg r$, for some $r, s \in$ Var, occur:

Ideally, if taxes are evaded $(p)$ a fine applies $(q)$. However, ideally, fines do not apply. Therefore, ideally, taxes are not evaded.

Indeed, such an argument can be encoded as follows:

$\begin{array}{lll}1 & (r \rightarrow s),(\neg s \rightarrow \neg r) \in g, \text { for some } g \in \mathcal{C} & \text { Hyp. } \\ 2 & \square(p \rightarrow q) & \text { Hyp. } \\ 3 & \square(p \rightarrow q) \rightarrow \square(\neg q \rightarrow \neg p) & 1 \times \mathrm{MD}_{\mathcal{C}} \\ 4 & \square(\neg q \rightarrow \neg p) & 2,3 \times \mathrm{MP} \\ 5 & \square \neg q & \text { Hyp. } \\ 6 & \square(\neg q \rightarrow \neg p) \rightarrow(\square \neg q \rightarrow \square \neg p) & \text { Axiom K } \\ 7 & \square \neg q \rightarrow \square \neg p & 4,6 \times \mathrm{MP} \\ 8 & \square \neg p & 5,7 \times \mathrm{MP}\end{array}$


An $\mathcal{L}$-model for $S_{\alpha 2}$ needs to satisfy all properties of $\mathcal{L}$-models for $S_{\alpha 1}$, plus the following:

- if $f(\phi \rightarrow \psi), f(\phi) \in h_{i}(w)$, then $f(\psi) \in h_{i}(w)$, for $i \in\{1,2\}$.

Definition 5.9 (System $S_{\alpha 3}$ ). The axiomatic basis of system $S_{\alpha 3}$ is specified by the following ordered list of deductive principles: $\left\langle\mathrm{MD}_{\mathcal{C}}, \mathrm{K}, \mathrm{T}^{*}, \stackrel{\circ}{4}, \mathrm{BR} 1\right\rangle$.

In $S_{\alpha 3}$ talk about iterated ideality or about iterated awfulness can be reduced to talk about simple ideality and simple awfulness, respectively, as the following derivation shows:

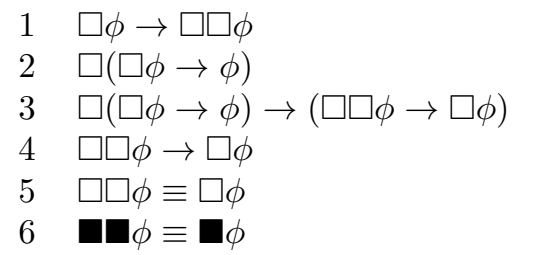

Axiom 4

Axiom T*

Axiom K

$2,3 \times$ MP

$1,4 \times P C$

$5 \times$ Mirror Images

An $\mathcal{L}$-model for $S_{\alpha 3}$ needs to satisfy all properties of $\mathcal{L}$-models for $S_{\alpha 2}$, plus the following:

- if $f(\phi) \in h_{1}(w)$, then $f(\square \phi) \in h_{1}(w)$;

- if $f(\phi) \in h_{2}(w)$, then $f(\boldsymbol{\square} \phi) \in h_{2}(w)$;

- $f(\square \phi \rightarrow \phi) \in h_{1}(w)$;

- $f(\boldsymbol{\square} \phi \rightarrow \phi) \in h_{2}(w)$.

The second group of systems that we are going to analyse ( $\beta$-systems) satisfy $\square$-monotony and -monotony over the set of theorems of the Propositional Calculus $(P C)$.

Definition 5.10 (System $S_{\beta 1}$ ). The axiomatic basis of system $S_{\beta 1}$ is specified by the following ordered list of deductive principles: $\left\langle\mathrm{RM}_{\mathrm{PC}}, \stackrel{\circ}{\mathrm{K}}, \mathrm{BR} 1\right\rangle$. 
An $\mathcal{L}$-model for $S_{\beta 1}$ needs to satisfy the following properties:

- $f(\mathfrak{c}) \in h_{1}(w)$ and $f(\neg \mathfrak{c}) \in h_{2}(w)$;

- if $\vdash_{P C} \phi \rightarrow \psi$, then $f(\phi) \in h_{i}(w)$ only if $f(\psi) \in h_{i}(w)$, for $i \in\{1,2\}$;

- if $f(\phi \rightarrow \psi), f(\phi) \in h_{i}(w)$, then $f(\psi) \in h_{i}(w)$, for $i \in\{1,2\}$.

Definition 5.11 (System $S_{\beta 2}$ ). The axiomatic basis of system $S_{\beta 2}$ is specified by the following ordered list of deductive principles: $\left\langle\mathrm{RM}_{\mathrm{PC}}, \mathrm{K}, \mathrm{T}^{*}, \stackrel{\circ}{4}, \mathrm{BR} 1\right\rangle$.

An $\mathcal{L}$-model for $S_{\beta 2}$ needs to satisfy all properties of $\mathcal{L}$-models for $S_{\beta 1}$, plus the following:

- if $f(\phi) \in h_{1}(w)$, then $f(\square \phi) \in h_{1}(w)$;

- if $f(\phi) \in h_{2}(w)$, then $f(\boldsymbol{\square} \phi) \in h_{2}(w)$;

- $f(\square \phi \rightarrow \phi) \in h_{1}(w)$;

- $f(\mathbf{\square} \phi \rightarrow \phi) \in h_{2}(w)$.

Definition 5.12 (System $S_{\beta 3}$ ). The axiomatic basis of system $S_{\beta 3}$ is specified by the following ordered list of deductive principles: $\left\langle\mathrm{RM}_{\mathrm{PC}}, \mathrm{K}, \mathrm{T}^{*}, 4, \stackrel{\circ}{\mathrm{D}}, \mathrm{BR} 1\right\rangle$.

An $\mathcal{L}$-model for $S_{\beta 3}$ needs to satisfy all properties of $\mathcal{L}$-models for $S_{\beta 2}$, plus the following:

- if $f(\phi) \in h_{i}(w)$, then $f(\neg \phi) \notin h_{i}(w)$, for $i \in\{1,2\}$.

In $\beta$-systems, as well as in all the $\alpha$-systems previously introduced, no formula of the form $m(\square \mathfrak{c} \wedge \boldsymbol{\square} \neg \mathfrak{c})$, where $m$ is a finite and non-empty sequence of occurrences of the operators $\square$ and/or $\mathbf{\square}$, is derivable. Moreover, already in $S_{\beta 1}$ the definition of an unconditional obligation $O \phi$ - that we obtained from the general schema $0 \mathrm{~b} *$ by equating $O \phi$ with $O(\phi / \top)-$ can be simplified as follows:

$\mathrm{Ob} *^{\prime} \quad O \phi=_{\text {def }} \diamond \top \wedge \square \phi \wedge(\diamond \top \rightarrow \neg \diamond \phi)$. 
Indeed, in $S_{\beta 1}$ the rule $\mathrm{RE}_{P C}$ is derivable. This entails that $\operatorname{RRPE}_{P C}$ is available. Due to the fact that $(\top \rightarrow \phi) \equiv \phi$ and $(\top \wedge \phi) \equiv \phi$ are derivable in $P C$, the intended simplification of $\mathrm{Ob} *$ to $\mathrm{Ob} *$ for unconditional obligations follows by applying $\operatorname{RRPE}_{P C}$ to the former.

Systems $S_{\beta 2}$ and $S_{\beta 3}$ allow for the already mentioned reduction of iterated ideality and iterated awfulness to simple ideality and simple awfulness, due to T* 4 and their mirror images. In system $S_{\beta 3}$ the definition of unconditional obligations can be further simplified so as to become identical with the one employed in [5], i.e., Ob2. Indeed, $\diamond \top$ and $\checkmark \top$ are theorems of $S_{\beta 3}$, as the following derivation shows (where we exploit the interdefinability of $\perp$ and $T$ and the definitions of $\diamond$ and $\diamond$ ):

$\begin{array}{lll}1 & \square \perp \rightarrow \neg \square \top & \text { Axiom D } \\ 2 & \perp \rightarrow \top & P C \\ 3 & \square \perp \rightarrow \square \top & 2 \times \mathrm{RM}_{P C} \\ 4 & \square \perp \rightarrow \perp & 1,3 \times P C \\ 5 & \neg \square \neg \top & 4 \times P C \\ 6 & \diamond \top & 5 \times \operatorname{Def}(\diamond) \\ 7 & \diamond \top & 6 \times \text { Mirror Images }\end{array}$

This derivation also points out that, as long as $P \phi$ is concerned, EPm* becomes equivalent to Pm1.

The third group of systems that we present here ( $\gamma$-systems) satisfy unrestricted $\square$-monotony and unrestricted $\mathbf{\square}$-monotony.

Definition 5.13 (System $S_{\gamma 1}$ ). The axiomatic basis of system $S_{\gamma 1}$ is specified by the following ordered list of deductive principles: $\left\langle\mathrm{RM}, \mathrm{K}, \mathrm{T}^{*}, 4, \stackrel{\circ}{\mathrm{D}}, \mathrm{BR} 1\right\rangle$.

An $\mathcal{L}$-model for $S_{\gamma 1}$ needs to satisfy all properties of $\mathcal{L}$-models for $S_{\beta 3}$, plus the following one, associated to rule RM (which entails the one associated to rule $\mathrm{RM}_{P C}$ in $\mathcal{L}$-models for $\left.S_{\beta 3}\right)$ :

- if $\vdash_{S_{\gamma 1}} \phi \rightarrow \psi$, then $f(\phi) \in h_{i}(w)$ only if $f(\psi) \in h_{i}(w)$, for $i \in\{1,2\}$.

In system $S_{\gamma 1}$ it is already possible to derive all formulas of the form $m(\square \mathfrak{c} \wedge \neg \mathfrak{c})$, where $m$ is a finite sequence of operators $\square$ and/or $\boldsymbol{\square}$. Indeed, we know that, if $m$ has length 0 , then $m(\square \mathfrak{c} \wedge \neg \neg)$ is BR1. Furthermore, the derivation below shows how to inductively move from a sequence $m$ of length $n$ to a sequence $m^{\prime}$ of length $n+1$. 


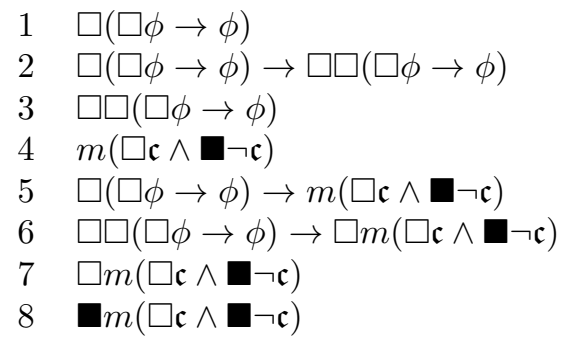

Axiom $\mathrm{T}^{*}$
Axiom 4
$1,2 \times \mathrm{MP}$
Induction hypothesis
$4 \times P C$
$5 \times \mathrm{RM}$
$3,6 \times \mathrm{MP}$
$7 \times$ Mirror Images

In the following systems, due to RM, $\mathrm{N}$ and their mirror images, the following rule and its mirror image are derivable:

$$
\text { RN } \quad \text { if } \vdash \phi \text {, then } \vdash \square \phi \text {. }
$$

Thus, these systems can be also interpreted in standard relational models for multimodal logic.

Definition 5.14 (System $S_{\gamma 2}$ ). The axiomatic basis of system $S_{\gamma 2}$ is specified by the following ordered list of deductive principles: $\left\langle\mathrm{RM}, \mathrm{N}, \mathrm{K}, \mathrm{T}^{*}, 4, \stackrel{\circ}{\mathrm{D}}\right.$, BR1 $>$.

An $\mathcal{L}$-model for $S_{\gamma 2}$ needs to satisfy all properties of $\mathcal{L}$-models for $S_{\gamma 1}$, plus the following:

- $f(\top) \in h_{i}(w)$, for $i \in\{1,2\}$.

Definition 5.15 (System $S_{\gamma 3}$ ). The axiomatic basis of system $S_{\gamma 3}$ is specified by the following ordered list of deductive principles: $\left\langle\mathrm{RM}, \mathrm{N}, \mathrm{K}, \mathrm{T}^{*}, 4, \mathrm{D}\right.$, BR2, BR1 $\rangle$.

An $\mathcal{L}$-model for $S_{\gamma 3}$ needs to satisfy all properties of $\mathcal{L}$-models for $S_{\gamma 2}$, plus the following:

- $f(\boldsymbol{\square} \phi) \in h_{1}(w)$ iff $f(\phi) \in h_{2}(w)$;

- $f(\square \phi) \in h_{2}(w)$ iff $f(\phi) \in h_{1}(w)$.

In system $S_{\gamma 3}$ the principle BR3 is derivable, since BR3 = mi(BR2). Due to this fact and the other axioms available (in particular, the interaction among $\mathrm{K}, \mathrm{T}^{*}, 4$ and their mirror images, as illustrated in a derivation above 
to get $\square \square \phi \equiv \square \phi$ and its mirror image), in $S_{\gamma 3}$ it is possible to reduce any finite and non-empty sequence $m$ of operators $\square$ and/or $\square$ to either a single occurrence of $\square$ or a single occurrence of $\boldsymbol{\square}$, and the schema $m(\phi) \rightarrow$ $(\square \phi \vee \square)$ is derivable. However, in principle, one can formulate systems in which only one among BR2 and BR3 is derivable, in order to represent an asymmetry between "awful ideality" and "ideal awfulness". ${ }^{11}$

\section{Representing Hohfeldian concepts}

We conclude this work with a concise discussion of a way in which basic Hohfeldian concepts, such as duty and right, can be represented within an extension of our bimodal language. First, we spend a few words on these concepts. The meaning of the terms "right" and "duty" has been debated at length over the last century - at least since the foundational work by W. N. Hohfeld in [9] and [10]. Hohfeld showed that there are four fundamental concepts that can be expressed by using the term "right" in the legal context: claim-right, privilege, power and immunity. Furthermore, he argued that rights and duties are to be regarded as correlatives: saying that a normative party $x$ has a duty towards a normative party $y$ to bring about $\phi$ is the same as saying that $y$ has a right against $x$ that $\phi$ be brought about. According to Hohfeld, two normative parties play a central role in descriptions of rights and duties, one of which can be labelled as the bearer (of the right/duty) and the other can be labelled as the counterpart (of the right/duty).

Hohfeldian concepts involving two normative parties can be captured via a variation of our bimodal language for normative ideality/awfulness including parametric operators, along the lines of [7]. The new language will be called $\mathcal{L}_{\text {Agt }}$. We take a set of agent-constants Agt and, for any $x, y \in \operatorname{Agt} \cup\{0\}$, there will be a pair of primitive modal operators $\square[x, y]$ and $\boldsymbol{\square}[x, y]$, in the place of the simple operators $\square$ and $\boldsymbol{\square}$. We will say that

\footnotetext{
${ }^{11}$ We stress that axiom $\mathrm{D}$ (as well as its mirror image) is independent from the rest of the axioms and rules for $S_{\gamma 3}$ (whence, from the rest of axioms and rules of any $\gamma$-system). Indeed, according to relational semantics for normal multimodal logic (see, e.g., [4]), RM, $\mathrm{N}, \mathrm{K}, \mathrm{T}^{*}, 4, \mathrm{BR} 2$ and their mirror images, together with BR1, are all valid in a a frame with a single world $w$ that has no access to itself (whence, to any world), whereas $\mathrm{D}$ (and its mirror image) can only be valid in frames where accessibility is a serial relation: for all $w$ there is $v$ s.t. $w$ has access to $v$.
} 
$x$ and $y$ in $\square[x, y]$ and $\boldsymbol{\square}[x, y]$ are parameters. In normal systems, these two kinds of operators read as follows:

- $\square[x, y] \phi$ means "in all normatively ideal situations $x$ brings about $\phi$ for/against $y "$;

- $[x, y] \phi$ means "in all normatively awful situations $x$ brings about $\phi$ for/against $y$ ".

The constant symbol 0 is used to denote "no agent"; for instance, we read $\square[x, 0] \phi$ as "in all normatively ideal situations $x$ brings about $\phi$ " (e.g., in all normatively ideal situations, Peter pays his debts/sees to it that his debts are paid); $\square[0, y] \phi$ as "in all normatively ideal situations $\phi$ is the case for/against $y$ " (e.g., in all normatively ideal situations, Peter's human rights are secured) and $\square[0,0] \phi$ as "in all normatively ideal situations $\phi$ is the case" (e.g., in all normatively ideal situations, good deeds are rewarded).

Logical systems over the extended language can be supplemented with the following two bridge-schemata (for any $x, y \in \mathrm{Agt}$ ):

BS1 $\square[x, y] \phi \rightarrow(\square[x, 0] \phi \wedge \square[0, y] \phi \wedge \square[0,0] \phi) ;$

BS2 $\mathbf{\square}[x, y] \phi \rightarrow(\boldsymbol{\square}[x, 0] \phi \wedge \boldsymbol{\square}[0, y] \phi \wedge \mathbf{\square}[0,0] \phi)$.

In normal systems, BS1 reads as follows: If in all normatively ideal situations $x$ brings about $\phi$ for/against $y$, then in all normatively ideal situations $x$ brings about $\phi$, in all normatively ideal situations $\phi$ is the case for/against $y$, and in all normatively ideal situations $\phi$ is the case. For example (and with a bit of simplification), if in all ideal situations Xavier $(x)$, the gardener, plants roses $(\phi)$ for Yvonne $(y)$ in her garden, then in all those situations he plants roses, she has the roses planted in her garden, and the roses are planted in her garden. BS2 reads analogously, but is concerned with normatively awful situations. Thanks to the schemata BS1 and $\mathrm{BS} 2$, if one considers systems where the formula $\diamond[x, y] \top \rightarrow \diamond[0,0] \top$ and its mirror image are derivable, then the definition $\mathrm{Ob} *$ allows one to get: $O[x, y] \phi \rightarrow O[0,0] \phi$. However, it is generally not possible to derive the converse implication.

Future research in this direction may explore the possibility of expressing more refined distinctions within Hohfeldian concepts involving two or more parties, as well as other related concepts (see, e.g., [18]). Moreover, it may aim at further assessing the advantages and disadvantages of the project of reducing normative concepts to the notions of normative ideality and normative awfulness, by identifying general expressive limits of the proposed language. 


\section{Contributions}

The contents of the article are the result of a joint research work of the two authors.

\section{References}

[1] A. Anderson, The formal analysis of normative systems, [in:] N. Rescher (ed.), The Logic of Decision and Action, University of Pittsburgh Press (1967), pp. 147-213.

[2] L. Åqvist, Deontic logic, [in:] Handbook of Philosophical Logic, vol. 8, Springer Netherlands (2002), pp. 147-264, DOI: https://doi.org/10.1007/ 978-94-010-0387-2_3.

[3] P. Cane, Responsibility in Law and Morality, Hart Publishing (2002).

[4] W. Carnielli, C. Pizzi, Modalities and Multimodalities, Springer (2008), DOI: https://doi.org/10.1007/978-1-4020-8590-1.

[5] M. de Boer, D. M. Gabbay, X. Parent, M. Slavkovic, Two dimensional Standard Deontic Logic [including a detailed analysis of the 1985 Jones-Pörn deontic logic system], Synthese, vol. 187 (2012), pp. 623-660, DOI: https://doi.org/10.1007/s11229-010-9866-4.

[6] S. Hansson, The varieties of permission, [in:] D. Gabbay, J. Horty, X. Parent, R. van der Meyden, L. van der Torre (eds.), Handbook of Deontic Logic and Normative Systems, College Publications (2013), pp. 195-240.

[7] H. Herrestad, C. Krogh, Obligations directed from bearers to counterparties, [in:] Proceedings of ICAIL 1995 (1995), pp. 210-218, DOI: https://doi. org/10.1145/222092.222243.

[8] R. Hilpinen, P. McNamara, Deontic logic: a historical survey and introduction, [in:] D. Gabbay, J. Horty, X. Parent, R. van der Meyden, L. van der Torre (eds.), Handbook of Deontic Logic and Normative Systems, College Publications (2013), pp. 3-136.

[9] W. N. Hohfeld, Some fundamental legal conceptions as applied in legal reasoning, Yale Law Journal, vol. 23 (1913), pp. 16-59.

[10] W. N. Hohfeld, Fundamental legal conceptions as applied in judicial reasoning, Yale Law Journal, vol. 26 (1917), pp. 710-770. 
[11] A. Jones, I. Pörn, Ideality, sub-ideality and deontic logic, Synthese, vol. 65 (1985), pp. 275-290, DOI: https://doi.org/10.1007/BF00869304.

[12] J. Jørgensen, Imperative and logic, Erkenntnis, vol. 7 (1937-1938), pp. 288296.

[13] T. Libal, M. Pascucci, Automated reasoning in normative detachment structures with ideal conditions, [in:] Proceedings of ICAIL 2019 (2019), pp. 63-72, DOI: https://doi.org/10.1145/3322640.3326707.

[14] H. Prakken, M. Sergot, Contrary to duty obligations, Studia Logica, vol. 57 (1996), pp. 91-105, DOI: https://doi.org/10.1007/BF00370671.

[15] W. V. O. Quine, On the nature of moral values, [in:] A. I. Goldman, J. Kim (eds.), Values and morals, Springer, Dordrecht (1978), pp. 37-45, DOI: https://doi.org/10.1007/978-94-015-7634-5_3.

[16] J. Raz, R. J. Wallace, The Practice of Value, Oxford University Press (2005), DOI: https://doi.org/10.1093/acprof:oso/9780199278466.001.0001.

[17] M. S. Schwartz, Universal moral values for corporate codes of ethics, Journal of Business Ethics, vol. 59 (2005), pp. 27-44, DOI: https: //doi.org/10.1007/s10551-005-3403-2.

[18] M. Sergot, Normative positions, [in:] D. Gabbay, J. Horty, X. Parent, R. van der Meyden, L. van der Torre (eds.), Handbook of Deontic Logic and Normative Systems, College Publications (2013), pp. 353-406.

[19] J. Sullins, Information Technology and Moral Values, [in:] E. N. Zalta (ed.), The Stanford Encyclopedia of Philosophy, spring 2021 ed., Metaphysics Research Lab, Stanford University (2021), https://plato.stanford. edu/archives/spr2021/entries/it-moral-values/.

\section{Daniela Glavaničová}

Comenius University in Bratislava

Faculty of Arts

Department of Logic and Methodology of Sciences

Bratislava, Slovak Republic

Slovak Academy of Sciences

Institute of Philosophy

Department of Analytic Philosophy

Bratislava, Slovak Republic

e-mail: daniela.glavanicova@gmail.com 


\section{Matteo Pascucci}

Slovak Academy of Sciences

Institute of Philosophy

Department of Analytic Philosophy

Bratislava, Slovak Republic

e-mail: matteopascucci.academia@gmail.com 